Gabriela Patricia Guijarro-Reinoso; Lorena Patricia Paredes-Lascano; Jessica Mariana Freire-Montesdeoca; Edgar Santiago Albarracín-López

http://dx.doi.org/10.35381/s.v.v4i8.984

\title{
Normativa AIEPI en niños de comunidades rurales tratados por enfermedad
} diarreica aguda

\section{AIEPI regulations in children from rural communities treated for acute diarrheal disease}

Gabriela Patricia Guijarro-Reinoso

gguijarro7840@uta.edu.ec

Postgrado de Medicina Familiar y Comunitaria, Facultad de Ciencias de la Salud, Universidad Técnica de Ambato, Ambato

Ecuador

https://orcid.org/0000-0002-7725-4514

Lorena Patricia Paredes-Lascano

patricialparedes@uta.edu.ec

Hospital General del Instituto Ecuatoriano de Seguridad Social. Ambato, Ambato

Ecuador

https://orcid.org/0000-0002-1029-9558

Jessica Mariana Freire-Montesdeoca

Jfreire0882@uta.edu.ec

Postgrado de Medicina Familiar y Comunitaria, Facultad de Ciencias de la Salud, Universidad Técnica de Ambato, Ambato

Ecuador

https://orcid.org/0000-0003-4997-9363

Edgar Santiago Albarracín-López

edgar.albarracin@iess.gob.ec

Hospital General del Instituto Ecuatoriano de Seguridad Social. Ambato, Ambato

Ecuador

https://orcid.org/0000-0002-8472-9661

Recepción: 15 de junio 2020

Revisado: 28 de julio 2020

Aprobación: 25 de agosto 2020

Publicación: 02 de septiembre 2020 
Gabriela Patricia Guijarro-Reinoso; Lorena Patricia Paredes-Lascano; Jessica Mariana Freire-Montesdeoca; Edgar Santiago Albarracín-López

\title{
RESUMEN
}

Objetivo: Evaluar el cumplimiento del AIEPI en la Diarrea en los niños de o a 5 años en un centro de salud. Materiales y métodos: se elaboró un checklist en base a la estrategia AIEPI. Se tomó las historias clínicas impares de manera aleatoria de enero - diciembre 2018. Resultados: En el centro de salud analizado se evidencia que el $76 \%$ de las historias utilizaron el AIEPI. En cuanto al tratamiento recibido destacan: la hidratación el $80 \%$ recibió suero oral. En el uso de antibióticos las 3/4 partes de las historias no utilizaron. En el $83 \%$ se prescribe signos de alarma y en el tiempo de recuperación existen 2 marcadas tendencias. Conclusión: Se concluye que el AIEPI se utiliza, pero amerita ciertos cambios.

Descriptores: Diarrea; diarrea infantil; epidemiología. (Fuente: DeCS2020).

\begin{abstract}
Objective: To evaluate compliance with $\mathrm{IMCl}$ in Diarrhea in children from or to 5 years old in a health center. Materials and methods: a checklist was prepared based on the $\mathrm{IMCl}$ strategy. Odd medical records were taken randomly from January - December 2018. Results: In the analyzed health center, it is evidenced that $76 \%$ of the records used the $\mathrm{IMCl}$. Regarding the treatment received, they stand out: hydration, $80 \%$ received oral serum. In the use of antibiotics $3 / 4$ parts of the stories were not used. In $83 \%$, warning signs are prescribed and in the recovery time there are 2 marked trends. Conclusion: It is concluded that IMCI is used, but it merits certain changes.
\end{abstract}

Descriptores: Diarrhea; diarrhea infantile; epidemiology. (Source: DeCS2020). 
Gabriela Patricia Guijarro-Reinoso; Lorena Patricia Paredes-Lascano; Jessica Mariana Freire-Montesdeoca; Edgar Santiago Albarracín-López

\section{INTRODUCCIÓN}

La enfermedad diarreica aguda sigue siendo una patología infecciosa y común en la edad pediátrica. ${ }^{1}$ En un grupo vulnerable (niños menores de 5 años) sigue teniendo importancia la patología por ser unos de los causales principales de morbi-mortalidad infantil. ${ }^{2}$ Según el Instituto Nacional de Estadística y Censos para el 2018 existieron 21241 ingresos hospitalarios por esta patología. ${ }^{2}$ La Organización Mundial de la salud y el Fondo de las Naciones Unidas crearon la normativa AIEPI con el fin descrito ${ }^{1}$ para el año 1996 Latinoamérica entre ellos el Ecuador asume esta normativa. La misma ha sufrido diversas modificaciones y actualizaciones de tal manera que en el país se implementó la última actualización entre el año 2016 y $2017 .^{3}$

La diarrea en menores de 5 años representa al $20 \%$ de la mortalidad a nivel mundial. ${ }^{1}$ Esto corrobora el Fondo Internacional de Emergencia de las Naciones Unidas para la Infancia que demostró la muerte de 43 niños por cada 1000 nacidos vivos por enfermedad diarreica. ${ }^{2}$ En América Latina especialmente en los países en vías de desarrollo aproximadamente $31 \%$ de las familias tienen pobreza extrema, considerándose como un factor de riesgo. ${ }^{3}$ Todo ello muestra que la diarrea en niños puede causar complicaciones agudas o crónicas que no les permiten un desarrollo óptimo y adecuado.

EI AIEPI nace en 1992 para reducir la mortalidad infantil producida por las principales enfermedades que se presentan entre los 0 y 5 años dentro de ellas la enfermedad diarreica. ${ }^{4}$ La implementación del AIEPI en el Ecuador forma parte del Modelo de Atención Integral del Sistema de Salud - FCl. Para ello en el año 1997 con un comité nacional se socializa la estrategia a nivel nacional y es utilizada desde entonces con las actualizaciones siendo la última en el 2018. ${ }^{5}$ Esta estrategia permite evaluar a los niños en base a una semaforización que indica el grado de riesgo y el tratamiento adecuado que debe ser administrado a dicho paciente como lo muestra de Sadai Mairena y Rizo Rodríguez. ${ }^{6}$ 
En la provincia de Tungurahua, así como en todo el Ecuador los niños menores de 5 años con enfermedad diarreica aguda deben ser atendidos en base al AIEPI en todos los niveles de atención haciéndose énfasis en el nivel primario. Además, esta atención es gratuita y se basa su diagnóstico en base a las necesidades de cada lugar tal como lo indica la estrategia.

Las indicaciones son claras para la enfermedad diarreica y se debe clasificar si tiene o no deshidratación, seguido de la administración de suero oral, no se recomienda el uso indiscriminado de antibióticos ni analgésicos mismo que se indica en situaciones específicas dadas por la misma estrategia. ${ }^{7}$ Además en la valoración de los signos de alarma (Letargia o inconciencia, ojos hundidos, no puede beber, signo de pliegue cutáneo) y en su control o próxima evaluación. ${ }^{8}$

Por lo anteriormente expuesto, la presente investigación tiene como objetivo valorar la utilización del AIEPI en la Diarrea Aguda en los niños de o a 5 años en un centro de salud.

\section{MÉTODO}

\section{Dimensión espacial de la investigación}

El sitio de estudio está ubicado en la provincia de Tungurahua cantón Ambato a una altura de 2713 msnm a 4465 msnm.

\section{Tipo de investigación}

Es un estudio cuantitativo observacional transversal y descriptivo.

\section{Muestra poblacional}

Se tomó las historias clínicas impares del registro médico con diagnóstico de diarrea y gastroenteritis de presunto origen infeccioso registradas en enero - diciembre 2018. Se obtiene mediante cálculo del tamaño conociendo el tamaño de la población un total de muestra de 307 historias clínicas mismas que fueron codificadas para que no sea factible su reconocimiento. 
Gabriela Patricia Guijarro-Reinoso; Lorena Patricia Paredes-Lascano; Jessica Mariana Freire-Montesdeoca; Edgar Santiago Albarracín-López

\section{Recopilación de la información}

Para la recolección de la muestra se elaboró un checklist en base a la estrategia AIEPI que abarca el diagnóstico y tratamiento de la enfermedad diarreica brindado dentro de un centro de salud (primer nivel) del área rural de una provincia céntrica del Ecuador.

\section{Análisis estadístico}

Se analizaron los datos en el sistema SPSS versión 25 y SPAD v56en.

\section{LIMITACIONES}

La principal encontrada fue la letra no legible, uso de corrector e información incompleta. Además, no es factible determinar si el examen físico del paciente se lo realizo adecuadamente, pudiendo ser este mal interpretado. El cumplimiento del AIEPI es tomado como un indicador de calidad, pero esto no significa que dicha estrategia haya sido aplicada al $100 \%$. Sin embargo, todas estas limitaciones son consideradas como incumplimiento a las directrices que brinda el AIEPI. 
Gabriela Patricia Guijarro-Reinoso; Lorena Patricia Paredes-Lascano; Jessica Mariana Freire-Montesdeoca; Edgar Santiago Albarracín-López

\section{RESULTADOS}

Se presentan los resultados de la investigación:

\section{Características de la población}

Tabla 1. Características de la Población

\begin{tabular}{|l|r|r|}
\hline \multicolumn{3}{|c|}{ EDAD_DEL_PACIENTE } \\
\hline \multicolumn{1}{|c|}{ Variable } & Frecuencia & Porcentaje \\
\hline 0 a 12 meses & 34 & $11 \%$ \\
\hline 13 a 24 meses & 96 & $31 \%$ \\
\hline 25 a 36 meses & 57 & $19 \%$ \\
\hline 37 a 48 meses & 60 & $19 \%$ \\
\hline 49 a 59 meses & 61 & $20 \%$ \\
\hline Total & 308 & $100 \%$ \\
\hline
\end{tabular}

Se determina que mayor atención por enfermedad diarreica se dio entre los 13 y 24 meses en el $31 \%$ seguido de los de 49 a 59 meses en el $20 \%$

Tabla 2. Sexo y etnia de los pacientes en estudio

\begin{tabular}{|l|r|r|}
\hline \multicolumn{3}{|c|}{ Sexo } \\
\hline Parámetro & Porcentaje & $\begin{array}{r}\text { Porcentaje } \\
\text { acumulado }\end{array}$ \\
\hline Masculino & $45,10 \%$ & $45,10 \%$ \\
\hline Femenino & $54,90 \%$ & $100 \%$ \\
\hline \multicolumn{3}{|c|}{ Etnia } \\
\hline Indígena & $83,80 \%$ & $83,80 \%$ \\
\hline Mestizo & $16,20 \%$ & $100 \%$ \\
\hline
\end{tabular}


Gabriela Patricia Guijarro-Reinoso; Lorena Patricia Paredes-Lascano; Jessica Mariana Freire-Montesdeoca; Edgar Santiago Albarracín-López

Resalta una relación 1:1 de hombres y mujeres y predomina la etnia indígena en el $83 \%$.

\section{Tratamiento}

En el centro de salud analizado se evidencia que el $76 \%$ de las historias utilizaron el AIEPI y apenas el $24 \%$ no lo aplicaron. Es importante señalar que el diagnostico en su gran mayoría lo realizaron por clínica sin solicitud de exámenes complementarios. En cuanto a los parámetros de tratamiento recibido destacan:

Tabla 3. Tipo de hidratación recibida.

\begin{tabular}{|l|r|r|}
\hline \multicolumn{3}{|c|}{ HIDRATACIÓN } \\
\hline & Frecuencia & Porcentaje \\
\hline LIQUIDO EN GENERAL & 8 & 2,6 \\
\hline NINGUNO & 26 & 8,4 \\
\hline SUERO CASERO & 11 & 3,6 \\
\hline SUERO ORAL & 247 & 80,2 \\
\hline VIA INTRAVENOSA & 16 & 5,2 \\
\hline Total & 308 & 100 \\
\hline
\end{tabular}

La hidratación el $80 \%$ recibió suero oral recomendado a nivel mundial como la más importante recomendación en diarrea de niños. 
Gabriela Patricia Guijarro-Reinoso; Lorena Patricia Paredes-Lascano; Jessica Mariana Freire-Montesdeoca; Edgar Santiago Albarracín-López

Tabla 4. Medicamentos utilizados en la enfermedad diarreica de niños menores de 5 años.

\begin{tabular}{|c|c|c|}
\hline \multicolumn{3}{|c|}{ ANTIBIÓTICO } \\
\hline & Frecuencia & Porcentaje \\
\hline NO & 267 & 86,7 \\
\hline SI & 41 & 13,3 \\
\hline Total & 308 & 100 \\
\hline \multicolumn{3}{|c|}{ ANALGÉSICO } \\
\hline & Frecuencia & Porcentaje \\
\hline $\mathrm{NO}$ & 137 & 44,5 \\
\hline SI & 171 & 55,5 \\
\hline Total & 308 & 100 \\
\hline \multicolumn{3}{|c|}{ ANTIPARASITARIO } \\
\hline & Frecuencia & Porcentaje \\
\hline $\mathrm{NO}$ & 251 & 81,5 \\
\hline SI & 57 & 18,5 \\
\hline Total & 308 & 100 \\
\hline \multicolumn{3}{|c|}{ ZINC } \\
\hline & Frecuencia & Porcentaje \\
\hline $\mathrm{NO}$ & 213 & 69,2 \\
\hline SI & 95 & 30,8 \\
\hline Total & 308 & 100 \\
\hline
\end{tabular}

En cuanto al uso de antibióticos las 3/4 partes de las historias no utilizaron, lo que no ocurre con los analgésicos porque más del $50 \%$ lo recibió encontrándose prescripción de paracetamol e ibuprofeno. En el uso de antibióticos se prescribe el Trimetoprim 
Gabriela Patricia Guijarro-Reinoso; Lorena Patricia Paredes-Lascano; Jessica Mariana Freire-Montesdeoca; Edgar Santiago Albarracín-López

Sulfametoxazol sin diagnóstico claro de disentería. No existe la prescripción de zinc el en $70 \%$ de los casos.

Tabla 5. Signos de alarma y tiempo de recuperación de los niños con diarrea.

\begin{tabular}{|c|c|c|}
\hline \multicolumn{3}{|c|}{ SE_INDICÓ_SIGNOS_DE_ALARMA } \\
\hline & Frecuencia & Porcentaje \\
\hline NO & 52 & 17 \\
\hline SI & 256 & 83 \\
\hline Total & 308 & 100 \\
\hline \multicolumn{3}{|c|}{ TIEMPO_DE_RECUPERACIÓN } \\
\hline & Frecuencia & Porcentaje \\
\hline 1-2 DIAS & 57 & 19 \\
\hline 3-4 DIAS & 116 & 38 \\
\hline 5-7 DIAS & 18 & 6 \\
\hline MAS DE 7 & 9 & 3 \\
\hline NO INDICA & 108 & 35 \\
\hline Total & 308 & 100 \\
\hline
\end{tabular}

Finalmente, en el tiempo de recuperación existen 2 marcadas tendencias la primera que los niños con diarrea de esta zona rural se recuperaron en 3 o 4 días reportados por consulta subsecuente o visita domiciliaria y la otra un poco alarmante pues no se determinó el tiempo de recuperación. 
Gabriela Patricia Guijarro-Reinoso; Lorena Patricia Paredes-Lascano; Jessica Mariana Freire-Montesdeoca; Edgar Santiago Albarracín-López

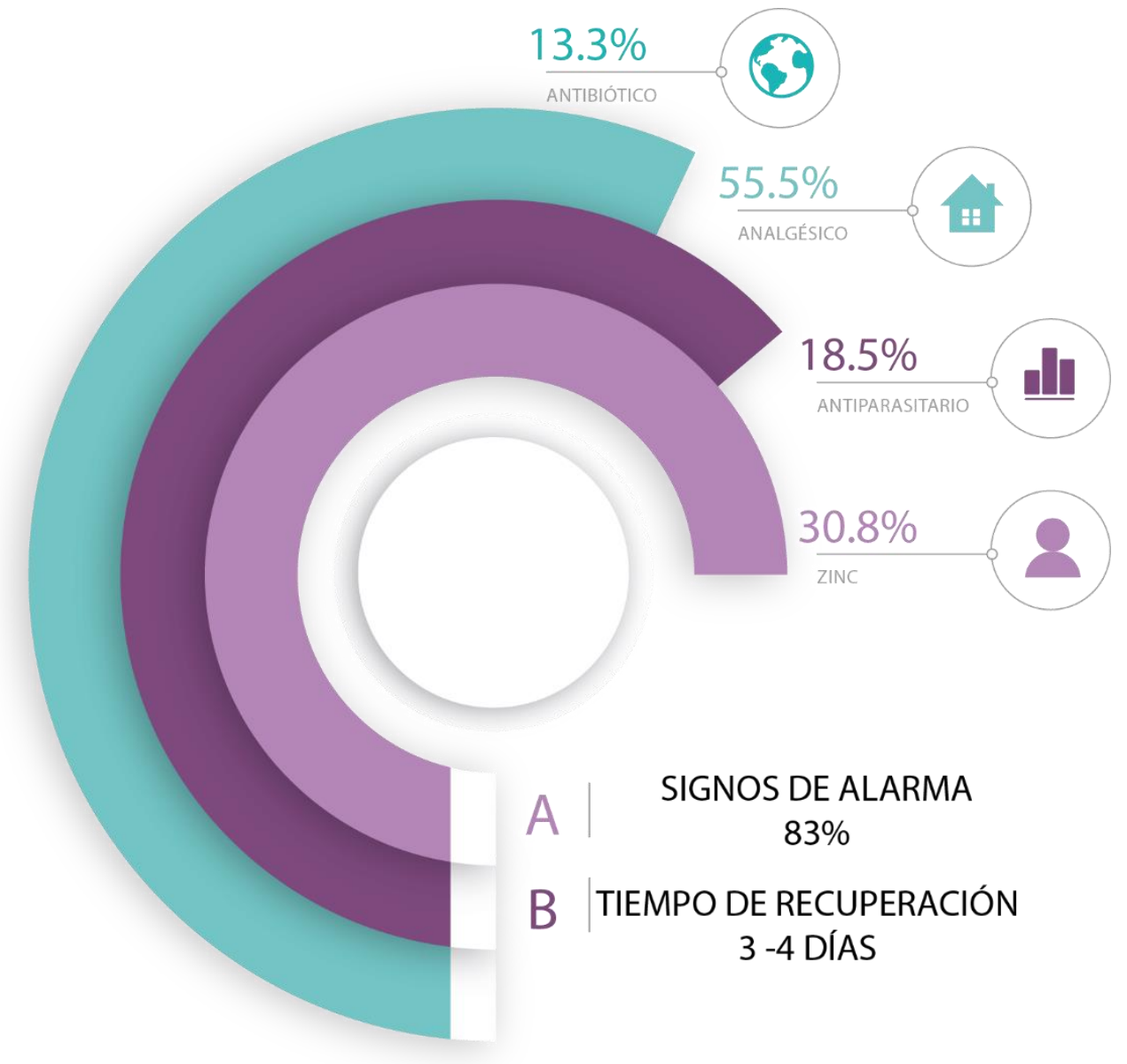

Figura 1. Esquematización de resultados finales de la investigación. Fuente: Los autores (2020).

\section{DISCUSIÓN}

La investigación pretendió determinar si el AIEPI es utilizado adecuadamente en la diarrea de los niños de una comunidad rural. Los resultados nos permiten mejorar la atención de un grupo prioritario y vulnerable dentro de una patología frecuente que no se ha podido erradicar. Los resultados contrastaran con el de Arciniegas y colaboradores $^{9}$ que determinaron en el 2016 en las enfermedades respiratorias y diarreicas el AIEPI se lo cumplió en el $95 \%$ con letra legible y clara. ${ }^{9}$ Esto llama la atención pues el porcentaje de $24 \%$ de incumplimiento es alto en base a estudios previos. 
A su vez se coincide con el diagnóstico clínico que se fortalece con el estudio de López Barón y colaboradores donde apenas el $15 \%$ de los resultados de los coproparasitarios solicitados fueron positivos para infección invasiva. ${ }^{10} \mathrm{El}$ suero oral como se indicó previamente es el pilar fundamental en el tratamiento de la diarrea esto lo afirma la Organización Mundial de la Salud y varios estudios como el de Carmona Jiménez en el 2017. ${ }^{11}$

En cuanto a la medicación prescrita es importante rescatar que no se usa indiscriminadamente los antibióticos como en otros estudios por ejemplo el realizado en Latinoamérica específicamente en Perú en el 2016 que se determinó en el 57\% de los niños recibieron antibióticos; similar al estudio realizado en Nicaragua que abordaron el $50 \%$ de uso de antibióticos; esto no ocurre en la parroquia estudiada actualmente. ${ }^{12,13}$

Al igual el uso de analgésico Gómez Toscano y la investigación actual determinan que en episodios febriles el uso de estos debe ser de manera individualizada. ${ }^{14}$ Dentro de estos los prescritos con mayor frecuencia es el paracetamol en sus diversas presentaciones y el Ibuprofeno medicamento que no consta en el AIEPI. Esto lo confirma Noroña Lucero ${ }^{15}$ en el 2019 que demuestra en el $20 \%$ se prescribe paracetamol y solo el 3\% ibuprofeno. ${ }^{15}$ En cuanto a la indicación del zinc Emir Vásquez ${ }^{13}$ noto que se administró en el $100 \%$ de los pacientes en contraste con el estudio actual donde la administración de este es nula o escasa. ${ }^{13}$

Los signos de alarma son importantes en las indicaciones del AIEPI pues de esto dependerá el tratamiento administrado o el cambio del mismo. Para ello Mites Portilla ${ }^{2}$ en el 2019 valoró los conocimientos de las madres de los signos de alarma arrojando el resultado del $52 \% .^{2}$ En cuanto a la evaluación de los signos de alarma Sernaque Izquierdo determino que el $73 \%$ de pacientes niños con diarrea no tuvieron signos de alarma. ${ }^{16}$

Finalmente, se debe hacer énfasis en el tiempo de recuperación, así como lo muestra Granera Salgado ${ }^{17}$ que el $69 \%, 27 \%$ y $4 \%$ de 169 niños con diarrea se recuperaron en 
1 a 3 días, de 4 a 7 días o más de 8 días respectivamente. Además, los niños internados el $40 \%$ permanecieron 48 horas.

Se debe realizar estudios cualitativos del personal y de los familiares con el fin de determinar específicamente las razones para incumplir con el AIEPI. Al valorar cualitativamente al personal se corroborará los resultados obtenidos y se podría buscar soluciones alternativas en base a cada paciente o de ser el caso solucionar los problemas administrativos o de desconocimiento.

Se debe reforzar en el uso de las recomendaciones que brinda el AIEPI actualizado como el zinc, la visita domiciliaria y consulta de seguimiento para evitar futuras complicaciones.

Es importante recordar que no solo se debe buscar el cumplimiento de los estándares de calidad de atención sino de dar un tratamiento adecuado y oportuno en base a cada paciente sin dejar de lado las recomendaciones del AIEPI. Finalmente, dentro de los niveles primarios de atención se debe preste mayor importancia al seguimiento y la indicación oportuna de los signos de alarma en caso de grupos vulnerables como los niños menores de 5 años.

\section{CONCLUSIÓN}

El estudio concluye que el AIEPI se utiliza, pero amerita ciertos cambios. Dentro de las indicaciones que si se realizan son la prescripción de suero oral, se indica signos de alarma. Los errores o los incumplimientos se dan en el uso de antibióticos y analgésicos, falta de seguimiento y la no prescripción de zinc. Es por ello que se da recomendaciones para la unidad de atención.

\section{CONFLICTO DE INTERÉS}

Los autores declaran que no tienen conflicto de interés en la publicación de este artículo. 
Gabriela Patricia Guijarro-Reinoso; Lorena Patricia Paredes-Lascano; Jessica Mariana Freire-Montesdeoca; Edgar Santiago Albarracín-López

\section{FINANCIAMIENTO}

Autofinanciado

\section{AGRADECIMIENTOS}

A la Universidad Técnica de Ambato, al Centro de Salud; por el apoyo en la realización de la investigación.

\section{REFERENCIAS}

1. Machado Núñez A. Utilidad del Hemograma y PCR para el inicio de antibióticos en niños menores de 5 años con Diarrea Aguda en el Hospital de Ventanilla en el período Enero 2017 - Julio 2017. [Usefulness of the CBC and CRP for the initiation of antibiotics in children under 5 years of age with Acute Diarrhea at the Hospital de Ventanilla in the period January 2017 - July 2017]. 2018. [Fecha de acceso: 6 junio 2019]. Recuperado de https://n9.cl/xbx6x

2. Carter, E., Bryce, J., Perin, J. et al. Harmful practices in the management of childhood diarrhea in low- and middle-income countries: a systematic review. BMC Public Health 15, 788 (2015). https://doi.org/10.1186/s12889-0152127-1

3. ENSANUT-ECU 2018. Encuesta Nacional de Salud y Nutrición. Ensanut [Internet]. 2018.1:47. [Fecha de acceso: 10 junio 2019]. Recuperado de https://n9.cl/dq1d

4. Thiam, S., Diène, A.N., Fuhrimann, S. et al. Prevalence of diarrhoea and risk factors among children under five years old in Mbour, Senegal: a crosssectional study. Infect Dis Poverty 6, 109 (2017). https://doi.org/10.1186/s40249-017-0323-1

5. Aldaz Sánchez A. Estrategia Para La Prevención De Enfermedades Diarreicas Aguda En Niños Menores De 3 Años En El Hospital Del Instituto De Seguridad Social Del Servicio De Pediatría De La Ciudad De Ambato En El Periodo Septiembre 2015 - Febrero 2016. [Strategy for the Prevention of Acute Diarrheal Diseases in Children Under 3 Years of Age at the Hospital of the Social Security Institute of the Pediatric Service of the City of Ambato in the period September 2015 - February 2016]. [Internet]. Universidad Regional Autónoma de los Andes. 2017. p. 69-71. [Fecha de acceso: 24 junio 2019]. Recuperado de https://n9.cl/ni74 
Gabriela Patricia Guijarro-Reinoso; Lorena Patricia Paredes-Lascano; Jessica Mariana Freire-Montesdeoca; Edgar Santiago Albarracín-López

6. Sadai Mairena R. Características en la atención al niño/a de 2 meses a 4 años de edad con la aplicación de la Estrategia AIEPI, Hospital Primario Jorge Navarro de Wiwili, Jinotega, Noviembre - Diciembre 2015. [Characteristics of child care from 2 months to 4 years of age with the application of the $I \mathrm{MCl}$ Strategy, Jorge Navarro de Wiwili Primary Hospital, Jinotega, November December 2015]. Univ Nac Autónoma Nicar [Internet]. 2015;33-62. [Fecha de acceso: 19 julio 2020]. Recuperado de https://repositorio.unan.edu.ni/4498/

7. Castillo IY, González YM, Gutiérrez N. Determinantes sociodemográficos de aplicación de prácticas clave AIEPI para prevenir enfermedades. área rural, Cartagena, Colombia. [Sociodemographic determinants of the application of key IMCl practices to prevent diseases. rural area, Cartagena, Colombia]. Hacia promoc salud [Internet]. 2016;7577(2):13-26.

8. Caluña López IR, Quintero Tanguila AE. Estrategia AIEPI: Signos de Peligro en Pacientes de 2 meses a 5 años, Centro de Salud Morete Puyo, octubre 2018 enero 2019. [IMCI Strategy: Danger Signs in Patients from 2 months to 5 years, Morete Puyo Health Center, October 2018 - January 2019]. Esc Super Politécnica Chimborazo [Internet]. 2019;37-45. [Fecha de acceso: 15 junio 2020]. Recuperado de https://n9.cl/fuwhc

9. Arciniegas HV, Ortiz AC, Marín OP, Valenzuela AB. Adherencia a la Guía de Práctica Clínica en la Atención Médica AIEPI en niños de 2 meses a 5 años, con Enfermedad Diarreica Aguda y Enfermedad Respiratoria Aguda, en la IPS Indígena del Municipio de Carlosama durante el periodo 2014-2 Y 2015-1 [Adherence to the Clinical Practice Guide in IMCI Medical Care in children from 2 months to 5 years, with Acute Diarrheal Disease and Acute Respiratory Disease, in the Indigenous IPS of the Municipality of Carlosama during the period 2014-2 and 2015-1]. 2016; 36-49. [Fecha de acceso: 19 junio 2020]. Recuperado de https://n9.cl/vodji

10. López Barón E, Morales Jiménez JC, Rodríguez Morales F. Análisis de costo oportunidad del uso de coproscópico en diarrea aguda en menores de 5 años.[Cost analysis - opportunity of the use of coproscopic in acute diarrhea in children under 5 years]. Rev Med [Internet]. 2016;24(2):66-73.

11. Yuquilema FS. Estrategias de aplicación del AIEPI comunitario para disminuir la morbilidad en los niños menores de 5 años en el Subcentro de Salud Yaruquies, Riobamba. [Community $\mathrm{IMCl}$ application strategies to reduce morbidity in children under 5 years of age in the Yaruquies Health Subcentre, Riobamba]. Univ Reg AUTÓNOMA LOS ANDES [Internet]. 2014. [Fecha de acceso: 14 junio 2019]. Recuperado de https://n9.cl/o4ng 
Gabriela Patricia Guijarro-Reinoso; Lorena Patricia Paredes-Lascano; Jessica Mariana Freire-Montesdeoca; Edgar Santiago Albarracín-López

12. Molina Rivera SH. Factores asociados a deshidratación en niños menores de 5 años con enfermedad diarreica aguda atendidos en el Hospital San José 20132015. [Factors associated with dehydration in children under 5 years of age with acute diarrheal disease treated at the San José Hospital 2013-2015]. Univ Ricardo Palma [Internet]. 2016;28-35. [Fecha de acceso: 28 junio 2020]. Recuperado de http://repositorio.urp.edu.pe/handle/urp/561

13. Vásquez GE. Cumplimiento del manejo terapéutico según AIEPI en los pacientes de 1 mes a 4 años de edad ingresados por diarrea en la sala de gastroenterología del hospital Alemán Nicaragüense en el período de Octubre - diciembre de 2016. [Compliance with therapeutic management according to $\mathrm{IMCl}$ in patients from 1 month to 4 years of age admitted for diarrhea in the gastroenterology ward of the German Nicaraguan hospital in the period of October - December 2016]. Univ Nac Autónoma Nicar [Internet]. 2017;29-32. [Fecha de acceso: 25 julio 2020]. Recuperado de https://repositorio.unan.edu.ni/7278/

14. Gómez - Toscano V. Use of antipyretics in pediatrics. Acta Pediatr Mex [Internet]. 2018;39(6):385-8. [Fecha de acceso: 07 julio 2020]. http://dx.doi.org/10.18233/APM39No6pp385-3881737

15. Noroña Lucero TE. Análisis de prescripciones de marzo, julio y diciembre del 2017 en menores de 5 años bajo lineamientos AIEPI en el Centro de Salud "Centro Histórico" [Analysis of prescriptions for March, July and December 2017 in children under 5 years of age under IMCI guidelines at the "Centro Histórico" Health Center]. [Internet]. 2019. 38-90. [Fecha de acceso: 07 julio 2020]. Recuperado de http://www.dspace.uce.edu.ec/handle/25000/18692

16. Sernaque Izquierdo FA. Gastroenteritis aguda en pacientes de 1 a 5 años en Hospital Martin Icaza de Babahoyo, periodo 2014-2015 [Acute gastroenteritis in patients aged 1 to 5 years at Hospital Martin Icaza de Babahoyo, period 20142015]. Univ Guayaquil [Internet]. 2017;28-40. [Fecha de acceso: 17 julio 2020]. Recuperado de http://repositorio.ug.edu.ec/handle/redug/32991

17. Granera Salgado TG. Caracterización clínico-epidemiológica de diarrea aguda con sospecha por rotavirus en menores de 5 años en Hospital Alemán Nicaragüense en 2015 y 2016 [Clinical-epidemiological characterization of acute diarrhea with suspected rotavirus in children under 5 years of age at Hospital Alemán Nicaragüense in 2015 and 2016]. Univ Nac Autónoma Nicar [Internet]. 2016;30-42. [Fecha de acceso: 21 julio 2020]. Recuperado de https://repositorio.unan.edu.ni/4469/ 
Revista Arbitrada Interdisciplinaria de Ciencias de la Salud. SALUD Y VIDA

Volumen 4. Número 8. Año 4. Julio - Diciembre 2020

Hecho el depósito de Ley: FA2016000010

ISSN: $2610-8038$

FUNDACIÓN KOINONIA (F.K).

Santa Ana de Coro, Venezuela.

Gabriela Patricia Guijarro-Reinoso; Lorena Patricia Paredes-Lascano; Jessica Mariana Freire-Montesdeoca; Edgar Santiago Albarracín-López

@2020 por los autores. Este artículo es de acceso abierto y distribuido según los términos y condiciones de la licencia Creative Commons Atribución-NoComercial-Compartirlgual 4.0 Internacional (CC BY-NC-SA 4.0) (https://creativecommons.org/licenses/by-nc-sa/4.0/). 